\title{
Los útiles óseos "poco elaborados" en el Paleolítico inferior y medio y su continuidad en el Paleolítico superior. Una revisión historiográfica
}

\author{
"Less-elaborate" bone tools during the Lower and Middle Paleolithic, and \\ its continuity during the Upper Paleolithic. An historiographical review
}

\author{
Millán Mozota Holgueras \\ Departamento de Antropología y Arqueología, IMF-CSIC \\ c/Egipcíaques 15, Barcelona (E-08001) \\ millanm@imf.csic.es
}

Recibido: 03-04-2013

Aceptado: 25-09-2013

\begin{abstract}
Resumen
Se presenta una revisión crítica de las evidencias de utillaje en materia ósea a lo largo del Paleolítico inferior y medio en Europa, así como de los denominados útiles "poco elaborados" en el Paleolítico superior y la Prehistoria reciente. Dicha revisión y sus resultados llevan a una serie de reflexiones relevantes para explicar la aparición de la llamada "industria ósea” en Europa, a inicios del Paleolítico superior. Y permiten sugerir algunas hipótesis sobre las posibles razones que subyacen a dicha aparición.
\end{abstract}

Palabras Clave: Hueso, Industria, Musteriense, Neandertal, Paleolitico, Utillaje.

\begin{abstract}
I present a critical review of the evidence on bone tools from Lower and Middle Paleolithic in Europe, and also on the so-called "less elaborated" tools from Upper Paleolithic and recent Prehistory. Such review and its results produce a number of relevant considerations to explain the appearance of the "bone industry" in Europe, in the beginnings of Upper Paleolithic. It also permits to suggest some hypotheses about the possible reasons behind this occurrence.
\end{abstract}

KeY words: Bone, Industry, Mousterian, Neanderthal, Paleolithic, Tools. 


\section{Introducción}

En las investigaciones sobre el Paleolítico, algunas visiones historiográficas (Noble y Davidson 1996) propusieron la ausencia de una verdadera "industria ósea" en Europa, antes de la llegada de los Humanos Anatómicamente Modernos (HAM) al inicio del Paleolítico superior. Dichas concepciones se insertaban en determinados modelos sobre la transición del Paleolítico medio al superior y la desaparición de los Neandertales. En particular, eran parte de las propuestas que explicaban ambos procesos históricos por una serie de incapacidades de las poblaciones neandertales frente los HAM (Mellars 2004).

En ese marco conceptual, la industria ósea se postuló como una de las aportaciones propias del kit intelectual de las poblaciones HAM que sustituyen a los últimos Neandertales en Europa.

Aunque buena parte de esos modelos han sido desestimados por las recientes investigaciones de los modos de vida en el Paleolítico medio (Ríos-Garaizar 2008, 2012), y de las capacidades simbólicas neandertales (Zilhao et al. 2010, Peresani et al. 2011), permanece como lugar común la ausencia de "industria ósea" sensu estricto, e incluso de utillaje en hueso, en el Paleolítico inferior y medio. En este trabajo se revisan las principales evidencias de uso de materiales óseos como instrumentos, desde los orígenes del género Homo, para comprobar la pertinencia de dicha idea, que es lugar común en nuestra disciplina.

Para la revisión del registro se hace necesario, en primer lugar, introducir una reflexión sobre si es apropiada la terminología que se ha utilizado. En concreto sobre los conceptos de "industria ósea" y "utillaje poco elaborado".

A partir de la base proporcionada por dicha reflexión, sigue una visión de síntesis de las numerosas evidencias de trabajo y uso del utillaje en hueso, en el Paleolítico inferior y medio. Estas evidencias están asociadas con poblaciones humanas que no son HAM, como H. habilis, $H$. heidelbergensis y $H$. neanderthalensis. En conjunto se recogen las evidencias más relevantes desde los homínidos africanos de hace 2 millones de años, hasta el final del Paleolítico medio.

Por último, y como elemento de contraste y reflexión, se extiende la revisión historiográfica a una serie de materiales óseos que no encajarían en la tradicional definición de industria ósea. Estos materiales provienen de contextos del Paleolítico superior y de grupos de cazadores-recolectores holocenos.

\section{El concepto de industria ósea y el utillaje poco elaborado}

En las últimas décadas se ha extendido el uso de la paráfrasis "poco elaborados/as" para referirse a conjuntos de utillaje del Paleolítico inferior y medio. Esos términos provienen de la historiografía paleolítica continental (Patou-Mathis 1999, Tartar 2003, Aguirre 2005/2006) y tienen su origen en la propia apariencia de los artefactos y en una cierta concepción sobre el cambio tecnológico en la Prehistoria. Los tipos específicos que se incluyen en esta categoría pueden variar mucho, y a menudo su denominación viene dada por las hipótesis aportadas sobre su función: retocadores, alisadores, cinceles o "pièces intermédiaires" (fr.), útiles cavadores, puntas, etc.

El hecho de adjetivar al utillaje del Paleolítico antiguo con la paráfrasis "poco elaborado" se basa en la comparación directa de esos materiales con industrias de elaboración o conformación del hueso, al modo del Paleolítico superior. Por tanto, la definición del utillaje se fundamenta en su "menor elaboración", con respecto a otro que es una "industria" - y de ese modo se conceptualiza, a su vez, como más avanzada.

En ese marco, las "industrias" documentadas en Europa desde el inicio del Paleolítico superior se asociaron con la superioridad cognitiva de las poblaciones de HAM (Mellars 1991). Y, consecuentemente, su ausencia en el Paleolítico medio (e inferior) se ha vinculado a incapacidades neandertales, en términos comparativos (Noble y Davidson 1996).

Patou-Mathis (1999) definió la industria ósea como repetición de gestos técnicos que llevan a la creación de piezas idénticas o muy próximas, en lo que constituye una definición ad hoc para el utillaje del Paleolítico superior. Esta formulación deja fuera a prácticamente todo el utillaje del Paleolítico antiguo -salvo quizás alguna excepción, como los materiales inferopaleolíticos del Lazio (Anzidei 2001).

Esa definición de industria ósea es problemática, porque excluye una parte importante de las evidencias de producción y uso de utensilios en hueso por parte de las sociedades de cazadoresrecolectores en general, y no sólo materiales del Paleolítico antiguo. Además, obliga a generar otro tipo de conceptos "accesorios", como la citada paráfrasis "poco elaborados".

No obstante, es cierto que la definición de industria ósea hecha por Patou-Mathis puede utilizarse si se cumplen dos condiciones: (1) comprender que la "industria ósea" se refiere a un concepto específico, y que es parte de una 
categoría estructural mayor, la del utillaje en hueso o los útiles óseos; y (2) evitar los prejuicios que arrastra con respecto a las capacidades cognitivas, de forma que no se identifique, de manera necesaria, la presencia de una "industria ósea" con una superioridad implícita de las poblaciones que la produjeron.

Volviendo al concepto de "útiles poco elaborados", dicha paráfrasis resulta muchas veces innecesaria, al provenir de una comparación apriorística con un utillaje del Paleolítico superior. Por ello, es recomendable evitar dicha adjetivación cuando resulte superflua a la descripción de los objetos y del contexto de estudio.

Sin embargo, cuando ambos materiales ("industria ósea" y "utillaje poco elaborado") conviven en el mismo marco de estudio, la terminología puede resultar eficaz. En concreto, la paráfrasis "poco elaborados" puede servir para referirse al utillaje que, según la definición de Patou-Mathis (1999) no entraría dentro del concepto de industria (utillaje conformado de forma seriada, y con una gran inversión de trabajo).

La propuesta de este autor, no obstante, es que los términos más adecuados son: útil (singular), útiles (plural) y utillaje (plural colectivo), haciendo referencia a la función, y a la naturaleza de instrumentos de trabajo. El concepto de industria queda, por tanto, incluido en el más general y coherente de utillaje, y sus rasgos característicos (seriación, repetición de gestos técnicos, inversión de trabajo, etc.) no son valores absolutos, o criterios clasificatorios, sino elementos integrables en la explicación: rasgos que reflejan las acciones y decisiones implicadas en la producción y gestión de los instrumentos de trabajo de una sociedad.

\section{Los orígenes del utillaje en hueso en el Paleolítico inferior}

La primera propuesta sobre útiles óseos ligados a los procesos de hominización los situaba entre los australopitecinos sudafricanos de hace unos dos millones de años. Se trata de la "industria osteodontoquerática" (Fig. 1), propuesta por Dart (1957). Este investigador retrató a los Australopithecus africanus como eficientes cazadores, que usaron instrumental de materias duras animales (huesos aguzados, garras, cuernas y dientes) para abatir y procesar a sus presas. Dichas presas de la caza serían después transportadas a sus lugares de habitación situados en cavidades kársticas.

Algunos trabajos expresaron dudas sobre la interpretación de Dart (Thenius 1973), pero fue un
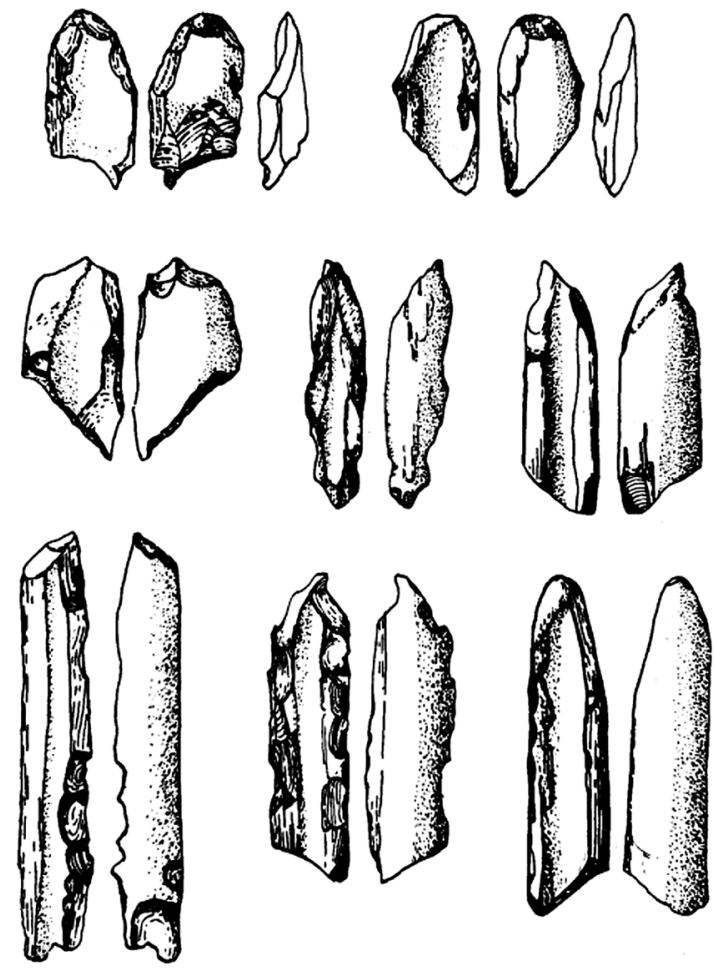

Figura 1.- Muestra de la denominada "industria osteodontoquerática" de Makapansgat. A partir de Lámina 5 en Dart (1960), dibujos originales de V. de Wet.

estudio de Brain (1981), con una crítica tafonómica de las evidencias, el que cambió la percepción de la "industria osteodontoquerática". Según Brain los restos óseos de las cavernas sudafricanas y los propios huesos de los homínidos eran el resultado fósil de la actividad de grandes felinos y otros carnívoros, que actuaron como principales agentes acumuladores. Las presas abatidas por esos depredadores incluían a los australopitecinos, y fueron transportadas a sus cubiles en cuevas, o bien a otros lugares (como los árboles sobre las dolinas de los karsts, que los leopardos utilizan como refugio temporal). En cuanto al utillaje y armamento propuesto por Dart, se trataría de pseudo-artefactos, producto de diversos procesos tafonómicos no antrópicos.

Las investigaciones sobre el utillaje en hueso más antiguo volvieron a la palestra en la primera década del S. XXI, con un trabajo de d'Errico y Blackwell (2003) que presentó el análisis de un conjunto de Swartkrans (Sudáfrica), de entre 1,8 y 2 millones de años de antigüedad. Se documentaron evidencias del uso del hueso y otras materias duras animales como utillaje. A partir de 
una metodología que incluye experimentación y el uso de colecciones etnográficas y paleontológicas, los investigadores presentaron varios útiles de hueso y asta (d'Errico y Blackwell 2003). Dichos artefactos habían sido primero modificados por abrasión, y después se usaron para cavar en tierra (suelos o termiteros). Un trabajo posterior de d'Errico (2007) resume las evidencias de las industrias óseas más antiguas, incluyendo Swartkrans y otros sitios africanos.

En Europa, desde los inicios de las disciplinas prehistóricas se ha propuesto la existencia de una serie de útiles de hueso del Paleolítico inferior. Con la llegada de la crítica tafonómica propuesta por Brain (1981), y por otros como Binford (1981), se comenzaron a estudiar o re-estudiar de manera sistemática dichas evidencias.

Esa crítica desde la tafonomía ha permitido determinar que existen numerosos agentes ( $y$ combinaciones de agentes) que pueden producir (d'Errico y Giacobini 1988) pseudo-artefactos, a menudo confundidos con útiles. Debido a la posibilidad de errar en la identificación macroscópica, la naturaleza tecnológica de los artefactos debe contrastarse con estudios de alcance suficiente: por lo general, usando microscopía óptica de altos aumentos (i. e. 100x-600x) o de barrido electrónico. Durante las últimas tres décadas, la crítica tafonómica entendida en estos términos ha llevado a desestimar buena parte de las evidencias que habían sido propuestas como útiles del Paleolítico inferior en Europa (d'Errico y Villa 1997).

Es importante señalar que la crítica y el análisis tafonómico de las evidencias dependen de técnicas y disciplinas en evolución. Por ello las conclusiones actuales no deben considerarse definitivas o inapelables, ya que en muchos casos se trata de hipótesis en proceso de corroboración o de resultados probables. Un caso que ilustra perfectamente estas dinámicas es el de la cabaña musteriense de huesos de mamut de Molodova I-N4 (aquí no se trata estrictamente de útiles óseos, sino de elementos constructivos). En este yacimiento se pueden ver cómo cambia la interpretación a la luz de estudios tafonómicos cada vez más detallados y precisos. En primer lugar los excavadores originales interpretaron la acumulación como una cabaña (Chernysh 1982), pero posteriormente fue desestimada como refugio construido por Kolen (1990). En un momento más reciente, sin embargo, un exhaustivo trabajo sobre los restos y su distribución espacial (Demay et al. 2012) ha vuelto a considerar que una cabaña es la explicación más razonable para la acumulación de huesos de mamut.
A continuación, se hace una breve referencia a los útiles óseos del Paleolítico inferior y medio que no han sido desestimados como tales. En estos casos, la crítica tafonómica respalda por el momento su naturaleza tecnológica.

Una parte importante de las evidencias de utillaje óseo del Paleolítico antiguo provienen de la península italiana. Se ha documentado la existencia de una producción mediante la talla por percusión en grandes fragmentos de diáfisis de elefante. Las evidencias provienen de los sitios de Castel di Guido (Radmilli y Boschian 1996), Fontana Ranuccio (Segre y Ascenzi 1984), Polledrara di Cecanibbio y Rebibbia-Casal de'Pazzi (Anzidei 2001), situados en la zona centro-occidental de Italia. Vértesszölös, en Hungría (Dobosi 2001), es un yacimiento inferopaleolítico, donde también se registra una producción ósea tallada sobre diáfisis de elefante. Para Anzidei et al. (2001) esto se integra en una estrategia de captación de materias primas que recurre al hueso para fabricar cierto tipo de herramientas, ante la escasez de litologías adecuadas a esos formatos. Costa (2010) considera ese recurso a la materia ósea como una cuestión de ubicuidad y facilidad de acceso frente a la, según su criterio, más compleja captación de materias primas líticas.

Los retocadores óseos forman una categoría de utillaje que, por lo general, se asocia con el Musteriense y el Paleolítico medio. No obstante, hay varias apariciones de este tipo de artefactos en el Paleolítico inferior europeo. Posiblemente, el más antiguo de estos ejemplos sea un retocador óseo hallado en Gran Dolina, en la Sierra de Atapuerca (España), junto con algunos huesos que pueden haber sido modificados por percusión (Rosell et al. 2011). El conjunto tiene una antigüedad de entre unos 300.000 y unos 350.000 años.

Dicha antigüedad podría ser superada por los "percutores" en hueso citados para Boxgrove, en Inglaterra (Roberts y Parfitt 1999), si bien la complejidad estratigráfica de dicho yacimiento, junto con la gran amplitud cronológica de los depósitos, no permiten precisar la cuestión.

En Francia, se han documentado retocadores óseos en el yacimiento de Orgnac (Moncel et al. 2005), datado en algo menos de 300.000 años. Para el OIS7 (h. 250-200.000 años) se han hallado en Biache-Siant-Vaast (Auguste 2002), y para el OIS 6 (h. 200-140.000 años) en la Cueva de Lazaret (Valensi 1996) y también (con una antigüedad mínima del OIS 5, que puede prolongarse hasta el OIS 6) en Velay (Raynal et al. 2005).

Como balance de las evidencias del Paleolítico inferior, se puede afirmar que existen suficientes pruebas de que se recurrió a la materia ósea, desde 
momentos muy tempranos de la evolución del género Homo. Esas evidencias incluyen el uso y la fabricación de útiles, tanto por técnicas de percusión, como por técnicas de abrasión.

\section{Las evidencias de trabajo y uso del hueso en el Paleolítico medio}

Para el Paleolítico medio, que en Europa a menudo se identifica con el Musteriense, existe un importante registro de utillaje óseo, que por diversas causas no ha sido estudiado con la suficiente profundidad.

Una de las menciones más antiguas de la presencia de utillaje óseo en el Paleolítico medio es la que hizo Daleau (1874), para la Grotte des Fées (Gironde, Francia). A principios del S. XX, Henri-Martin estudió los restos óseos de La Quina y determinó la existencia de un tipo concreto de útil: objetos en esquirlas diafisarias de ungulado, posiblemente utilizados para retocar las piezas líticas musterienses (Henri-Martin 1906).

En aquellas primeras décadas del S. XX, esos retocadores recogidos por Henri-Martín, junto con objetos similares provenientes de otros lugares (Rebiéres), se publicaron casi siempre como "yunques" o percutores durmientes (Baudoin 1906). Paralelamente, G. de Mortillet y H. de Mortillet (1910) definieron -para el Solutrense- el tipo "compresor". Se trataba, en ese caso, de un útil de hueso o asta asociado al retoque por presión. De manera indirecta, esa definición motivó que las esquirlas diafisarias con impresiones, descritas por Henri-Martin para el Paleolítico medio, se denominaran durante mucho tiempo "compresores-retocadores".

Breuil y Obermaier (1914) señalan la presencia de algunos huesos utilizados en los niveles musterienses de El Castillo (Cantabria). Passemard (1922) cita diáfisis con impresiones provenientes de Isturitz (Pyrénées-Atlantiques). Siret experimenta con el uso del hueso en percusión sobre útiles líticos. Utiliza esquirlas diafisarias de grandes ungulados para retocar piezas similares a las musterienses. Su investigación concluye que los fragmentos diafisarios con impresiones de los yacimientos son retocadores para el trabajo del sílex, y no elementos pasivos (Siret 1925).

En la década de 1930, se citan como "compresores" una serie de fragmentos óseos musterienses de La Ferrasie (Peyrony 1934).

Los estudios soviéticos de traceología, que llegan a Europa occidental en los años cincuenta, supusieron un impulso decisivo para la expansión del método experimental y del estudio de huellas de uso. Los trabajos de Semenov tuvieron una influencia directa en el análisis del utillaje óseo musteriense, y permitieron consolidar las herramientas metodológicas de estudio ya existentes (Semenov 1956).

A principios de los años sesenta, F. Bordes cita en sus listas tipológicas los compresores óseos solutrenses, pero no considera que respondan al mismo tipo de utilización que las esquirlas diafisarias musterienses con impresiones (Bordes 1961: 108).

La confusión o variabilidad terminológica al referirse a los retocadores musterienses se va a mantener hasta más allá de los años 60 . Durante esa década los avances de la disciplina, y las visiones de síntesis, dan una idea más clara de la naturaleza de dichos objetos. A partir de entonces se caracteriza la mayor parte de aquellos objetos musterienses como "retocadores". Es decir, elementos de percusión utilizados para el retoque de útiles de piedra (Patou-Mathis 2002). Sin embargo, una denominación mixta ("compresoresretocadores") se ha mantenido hasta épocas relativamente recientes (Barandiarán 1987).

Un trabajo de síntesis para el continente europeo (Taute 1965) recogió una gran recopilación de retocadores en materias óseas (hueso, dientes $\mathrm{y}$ asta), con una perspectiva cronológica amplia, que va del Paleolítico antiguo hasta el Neolítico. Su trabajo incluye la publicación de retocadores de Paleolítico medio, encontrados en varios yacimientos alemanes. Para el grueso de los retocadores óseos (esquirlas diafisarias y epifisarias, con huellas impresas), Taute concluye que se trata de un utillaje para el retoque lítico, por percusión.

Desde la década de los años setenta, se constata una presencia cada vez más habitual y abundante (en cuanto a efectivos por yacimiento), de retocadores óseos en niveles musterienses. Se documentan y citan en yacimientos franceses, como Bois-Roche en Charente (Vincent 1987); Y, una vez más, en Isturitz (Leroy-Prost 1974), aunque se utiliza el término "compresores".

Cada vez con mayor frecuencia, aparecen retocadores sobre esquirlas diafisarias en yacimientos de Paleolítico medio de otras zonas del continente europeo. En Kulna, Moravia (Valoch 1988), en el Abrigo Tagliente, en Verona (Leonardi 1979), en Bacho-Kiro, Bulgaria (Kozlowski 1982) y Peña Miel, en La Rioja (Barandiarán 1987).

El trabajo de Barandiarán (1987) sobre los "compresores-retocadores" de Peña Miel es el primer esfuerzo sistemático, en el ámbito de la Península Ibérica, para estudiar con una metodología específica un conjunto de utillaje óseo 
musteriense. Barandiarán estudia 67 retocadores, que provienen del nivel G de Peña Miel. Su trabajo analiza el origen anatómico, las dimensiones de los soportes y las características de las huellas de uso. En sus conclusiones, Barandiarán (1987) señala la vinculación directa de los retocadores con el trabajo de la piedra. Una revisión reciente de este conjunto (Mozota 2012) ha confirmado esa afirmación y ha permitido localizar 95 retocadores más entre los restos de fauna del Nivel G de Peña Miel.

Los trabajos experimentales también continúan sucediéndose a lo largo de estas décadas. Se trata de estudiar la relación de los percutores y el utillaje lítico, así como aspectos más generales de la modificación y fragmentación de la materia ósea (Dauvois 1974, Vincent 1985).

La constatación de la presencia de retocadores en muchos yacimientos musterienses discurre de manera paralela a la crítica tafonómica de los posibles artefactos óseos propuestos para el Paleolítico medio. En la Región Cantábrica, se suceden las propuestas (Freeman 1971) y críticas (Binford 1983, Straus 1992) para el yacimiento de Cueva Morín (Cantabria). Freeman presenta una serie de artefactos de hueso de origen antrópico del nivel 17 (Musteriense), pero Binford (1983) considera que se trata de pseudo-artefactos, y reinterpreta las hipotéticas huellas de fabricación como producto de la fractura del hueso para obtener el tuétano. Martínez-Moreno (2005) abunda en esta opinión señalando por otro lado la posible presencia no confirmada de retocadores óseos. A ese respecto, en las publicaciones sobre Morín (Freeman 1971) se citan 63 huesos descritos como fragmentos de diáfisis con "machacamientos" en los extremos. De ese conjunto, al menos una parte de los mismos son retocadores óseos (Mozota 2012).

A finales de los ochenta varios trabajos regresan a la crítica tafonómica de los materiales del Paleolítico medio. Se hace hincapié en la naturaleza de pseudo-artefactos de muchos hallazgos considerados hasta entonces útiles óseos del Musteriense. Se introduce el uso de la microscopía electrónica (Microscopio Electrónico de Barrido MEB) y de las réplicas en resinas sintéticas de las huellas de uso (d'Errico y Giacobini 1988).

De modo paralelo, se presentan nuevas evidencias de utillaje óseo para el Musteriense, que superan una crítica tafonómica cada vez más rigurosa. Algunos casos bien documentados de materiales óseos musterienses provienen del nivel VIII de Grotte Vaufrey, Dordogne (Vincent 1988).

En los años 90 hubo un importante desarrollo de las investigaciones sobre el utillaje óseo en el
Musteriense europeo. A principios de la década, Chase (1990) estudia una muestra de 32 huesos utilizados provenientes de La Quina, y concluye que son esquirlas utilizadas como retocadores. Dicha conclusión tiene cierto apoyo experimental, con un valor replicativo, para constatar la naturaleza de las huellas.

Una tesis doctoral de comienzos de los años noventa recoge una síntesis del utillaje óseo del Paleolítico medio (Vincent 1993), proponiendo nuevos enfoques metodológicos, que recogen los avances en arqueozoología y tafonomía de los últimos años.

En 1996 Sáenz de Buruaga presenta un trabajo que recoge evidencias de utillaje en hueso para el Musteriense y el Auriñaciense de la Cueva de Gatzarria y de Abeilles (Francia). Además de constatar la presencia de retocadores, este investigador apunta la posible existencia de huesos modificados por percusión (es decir, tallados o retocados) en los niveles estudiados.

En 1999 un trabajo sobre el utillaje óseo de Salzgitter-Lebenstedt (Alemania), aportó evidencias de la elaboración de útiles de hueso, con técnicas relativamente complejas (Gaudzinski 1999).

Ese yacimiento de Paleolítico medio cuenta con dos niveles sucesivos, de sedimentos fluviales, que proporcionaron 29 útiles de hueso de mamut. A partir del análisis tafonómico y arqueozoológico se plantea el aprovechamiento de una materia prima obtenida del carroñeo. Las fracturas en espiral de los huesos muestran que se astillaron en fresco, usando elementos de percusión masiva, lo que apunta a que se aprovecharon las carcasas poco después de la muerte de los animales. Del conjunto de utillaje en hueso, destacan 20 costillas de mamut, trabajadas a partir de una cadena operativa (Fig. 2) bastante compleja, con una técnica refinada para trabajar materiales óseos (Gaudzinski 1999). La importancia de SalzgitterLebenstedt radica en que dicha cadena operativa y las técnicas aplicadas permiten refutar la ausencia de un utillaje óseo complejo y estandarizado en el Paleolítico medio.

A lo largo de los años noventa, y hasta la actualidad, se constata la presencia de retocadores de hueso en numerosos yacimientos de toda Europa, que pertenecen a cronologías de Paleolítico medio. En la Europa centro-oriental, se citan retocadores de hueso en Prolom II (Stephanchuk 1993) y Kabazi V (Patou-Mathis 2007) en Crimea, Barakaevskaia, en el Cáucaso (Filipov y Lioubine 1993) y Vindija en Croacia (Ahern et al. 2004). La zona mediterránea queda representada por Fumane (Jéquier et al. 2012), Tagliente y San Bernardino (Malerba y Giacobini 1996) en Italia, Arrillor 
(Bermúdez de Castro y Sáenz de Buruaga 1999), Covalejos (Sanguino y Montes 2005), Axlor (González Urquijo et al. 2005, Mozota 2009) (Fig. 3), Prado Vargas y Valdegoba (Diez FernándezLomana y Navazo 2005) en España, y Figueira Brava (Antunes 2000) en Portugal.

En Francia, destacan los yacimientos de Lazaret (Valensi 1996), Chez-Pinaud (Beauval 2004), Artenac (Armand y Delagnes 1998), Sainte-Anne 1 en Polignac (Raynal et al 2010) y Abri Laborde/ Baume-Vallée (Raynal et al. 2005). Además, se publica el primer caso conocido de un retocador sobre hueso humano (Verna y d'Errico 2010), que proviene de los niveles musterienses de La Quina.

Fuera de Europa, se documentan retocadores en un contexto de Middle Stone Age (MSA), en Blombos Cave, Sudáfrica (Henshilwood et al. 2001); y en el Paleolítico medio de Siria en Ummel-Tlel (Boeda et al. 1998), y de Marruecos en El Harhoura (Michel et al. 2009).

Un trabajo sobre los procesos de talla y retoque de las raederas Quina en Francia (Bourguignon 2001) da cuenta de la realización de un importante programa experimental que incluye el uso de retocadores de piedra y hueso. En dicho trabajo se asocia el uso de retocadores de hueso (dentro de la categoría de los percutores "blandos") con una fase del retoque de los filos Quina. La autora señala que las huellas de uso en los retocadores experimentales son idénticas a las observadas en algunos materiales arqueológicos musterienses (Bourguignon 2001: 37).

En 2002 se publicó un trabajo de síntesis sobre retocadores, coordinado por M. Patou-Mathis, en el marco de una serie de cuadernos (fr. cahiers) denominados "Fichas Tipológicas de Industrias óseas prehistóricas" (Patou-Mathis 2002). Ese estudio de síntesis recoge toda una serie de colecciones de retocadores, principalmente Musterienses, en forma de fichas tipológicas estandarizadas.

Dicho trabajo aporta una metodología de estudio, basada en la tipificación de los diferentes aspectos morfológicos, tecnológicos y tafonómicos. No obstante, debe tomarse con muchas reservas la afirmación hecha en ese trabajo (Auguste 2002) de que se pueden considerar los retocadores como una "especifidad" neandertal. Por el contrario, los retocadores están presentes también en el Paleolítico superior, en otros contextos de cronología holocena, y también en la MSA (Middle Stone Age) africana.

Un caso de interés, entre las fichas tipológicas, son los retocadores en extremos proximales de húmeros de grandes ungulados (Valensi 2002). Estos útiles provienen de los niveles musterienses

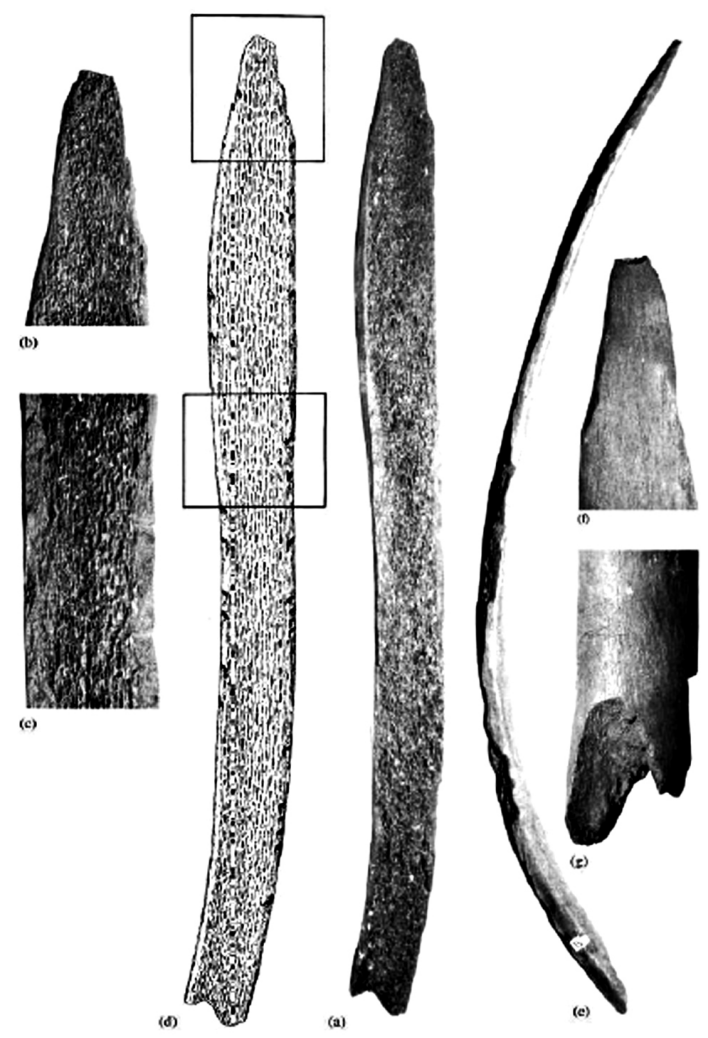

Figura 2.- Costilla de Mammuthus primigenius procesada y modificada de forma sistemática, proveniente del yacimiento de Salzgitter-Lebenstedt. A partir de Figura 11 en Gaudzinski (1999).

de La Quina, y su importancia reside en dos factores: (1) no son fragmentos de diáfisis, sino extremos de la región metafisaria del húmero; y (2) se documenta un proceso de conformación del soporte, mediante una sucesión de fracturas, efectuadas con un percutor masivo, y seguidas de una abrasión de dicha zona. Es decir, una importante inversión de trabajo, y una técnica avanzada de conformación del utillaje.

En un trabajo ya citado (Martínez-Moreno 2005) se han revisado también útiles "poco elaborados" hallados en las excavaciones antiguas de Lezetxiki. Los huesos hendidos, propuestos como útiles, se desestiman, y los estigmas se atribuyen al efecto de "microflaking" producido con el colapso de la diáfisis, cuando se fractura. También se caracterizan como pseudo-artefactos huesos modificados por abrasión o perforación, atribuyendo las modificaciones al efecto de agentes tafonómicos como el arrastre en seco, el efecto del agua, o los dientes de carnívoros.

Por otra parte, en las excavaciones recientes de Axlor (González Urquijo et al. 2005) se han 
recogido una serie de útiles en hueso provenientes de varios niveles de la secuencia musteriense (Mozota 2007, 2008). Entre esos materiales destacan una serie de costillas y diáfisis (Fig. 4) de grandes ungulados utilizadas en tareas variadas (Mozota 2007, 2012), así como algunos cinceles (Fig. 5) usados para el trabajo de materiales de cierta dureza (i.e. hueso, asta o madera). Estos últimos muestran similitudes con el tipo "pièces intermediaires" (fr.) descrito por Tartar (2003).

La evidencia del Paleolítico medio apunta por tanto a un papel muy importante de los retocadores óseos, que son útiles de producción (para la conformación y mantenimiento del utillaje en piedra) y forman parte del kit técnico más habitual encontrado en los yacimientos de estas sociedades humanas.

Por otra parte, aparecen otro tipo de útiles también (Mozota 2012), y en ciertos momentos y lugares del Paleolítico medio (Gaudzinski 1999, Valensi 2002) se documenta el trabajo del hueso mediante cadenas operativas complejas. Esos procesos no son idénticos a los del Paleolítico superior, pero sí resultan similares en términos de complejidad y planificación.

\section{Pervivencia de retocadores y del utillaje "poco elaborado" en cronologías posteriores al Paleolítico medio}

Los útiles óseos que se han dado en llamar "poco elaborados" (como los retocadores en esquirlas diafisarias) no son una especifidad del Paleolítico medio, sino que están presentes en cronologías posteriores y en muy diversos ámbitos geográficos. Sin embargo, a menudo han pasado desapercibidos para los investigadores.

Para la Europa del Paleolítico superior inicial, hay noticia de importantes conjuntos de retocadores óseos en yacimientos del Protoauriñaciense y el Auriñaciense, junto con algunos ejemplos en contextos chatelperronienses.

Hay un conjunto destacado en la Cueva de Gatzarria: A partir del material figurado por Sáenz de Buruaga (1987) resulta claro que se documentan al menos 25 retocadores y un posible cincel de hueso en el nivel Cjn2 (Protoauriñaciense). En el Auriñaciense antiguo (Nivel Cbi-Cbf) habría seis posibles cinceles, un posible hueso retocado, y 15 retocadores óseos; y en el Auriñaciense Evolucionado (Nivel $\mathrm{Cb}$ ), aparecen dos posibles cinceles y ocho retocadores óseos.

Junto a estos materiales se figuran también otros posibles útiles en hueso (Sáenz de Buruaga 1987) de Gatzarria, que parecen a priori algo más dudosos. Algunos son presentados como huesos retocados, pero su apariencia general sugiere que se trata de huesos con saltados y desconchados, relacionados con la fractura y colapso de la diáfisis. Otros huesos tienen marcas incisas o posibles huellas de impacto, y aunque se sugiere que pudieron ser utilizados como yunques, es difícil corroborarlo a partir de la información disponible.

Otra secuencia que ha dado retocadores óseos en varios momentos del Auriñaciense es Isturitz (Leroy-Prost 1974). Dichos útiles son muy similares a los musterienses (Schwab 2003). Por otro lado, en varios yacimientos de Charente de adscripción auriñaciense se han recogido caninos de úrsidos, con huellas de uso que se describen como similares a las marcas de los retocadores en esquirlas diafisarias (Leroy-Prost 2002). Ese mismo tipo de útil, en canino de oso, se documenta en Vogelherd, con seis ejemplares en un contexto también Auriñaciense (Leroy-Prost 2002). Por último, en los niveles del Auriñaciense antiguo de la Grotte des Hyènes (Brassempouy) se ha estudiado un utillaje (Fig. 6) muy similar al hallado en contextos musterienses, que incluye retocadores, cinceles (fr. pièces intermediaires"), punzones y un "alisador" (Tartar 2003).

En el Chatelperroniense de Arcy-sur-Cure, en la Grotte du Bison se ha constatado la presencia de dos retocadores, en esquirlas diafisarias de tibia de reno y metapodio de caballo (David et al. 2005).

Como se afirma en los trabajos de Tartar (2003, 2012), esas evidencias, denominadas "poco elaboradas", han sido por lo general ignoradas por los investigadores. Y, a pesar de su papel técnico $\mathrm{y}$ económico en las actividades de subsistencia, no han contribuido a las explicaciones de la organización o el desarrollo histórico de los grupos humanos.

Ya avanzado el Paleolítico superior, para el Solutrense de Laugerie-Haute (Dordoña) se han hallado dos retocadores sobre dientes de caballo, que, según los investigadores que los han estudiado (Castel y Madelaine 2006) son similares a los documentados en el Musteriense y el Auriñaciense. Dicha constatación hace que insistan en la necesidad de buscar ese tipo de materiales, que a menudo pueden pasar desapercibidos, en los conjuntos de Paleolítico superior (Castel y Madelaine 2006). También para el Solutrense, en Le Petit Cloup Barrat (Cabrerets) se han encontrado dos retocadores en esquirlas diafisarias de ungulados de talla media (Castel et al. 2006).

Para el Magdaleniense, en la última etapa del Paleolítico superior, se han citado retocadores óseos en el Rhin (Tinnes 2001), en los yacimientos 


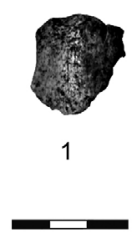

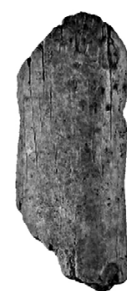

2

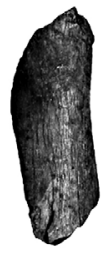

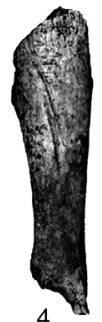
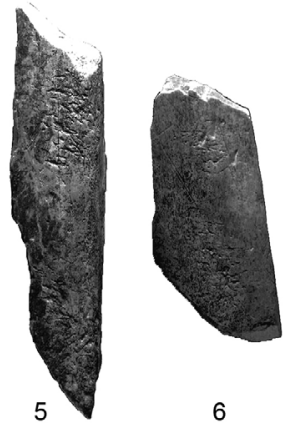

6
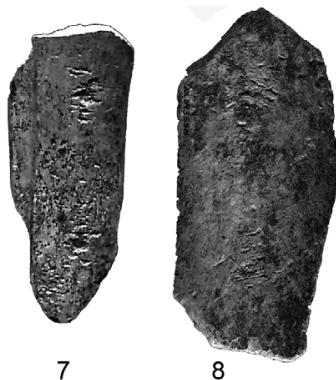

Figura 3.- Fragmento de retocador óseo (1) y retocadores óseos del Nivel M (2-4) y Nivel D (5-8) de Axlor (Dima, Bizkaia); A partir de Lámina I en Mozota (2009) y Figura IV-11 en Mozota (2012).
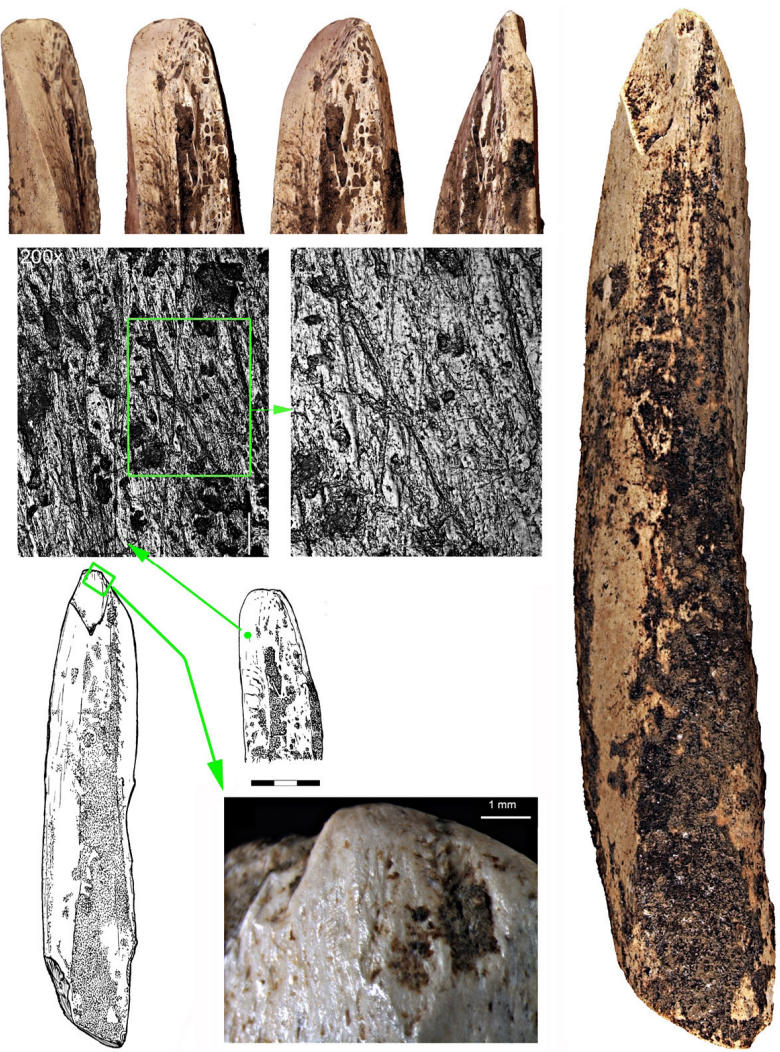

Figura 4.- Fragmento diafisario de tibia de gran bóvido utilizado para trabajar una materia resistente pero de cierta flexibilidad (e. g. corteza) proveniente de Axlor Nivel B. A partir de Figura 3 en Mozota (2007) y Figura VI-18 en Mozota (2012). 

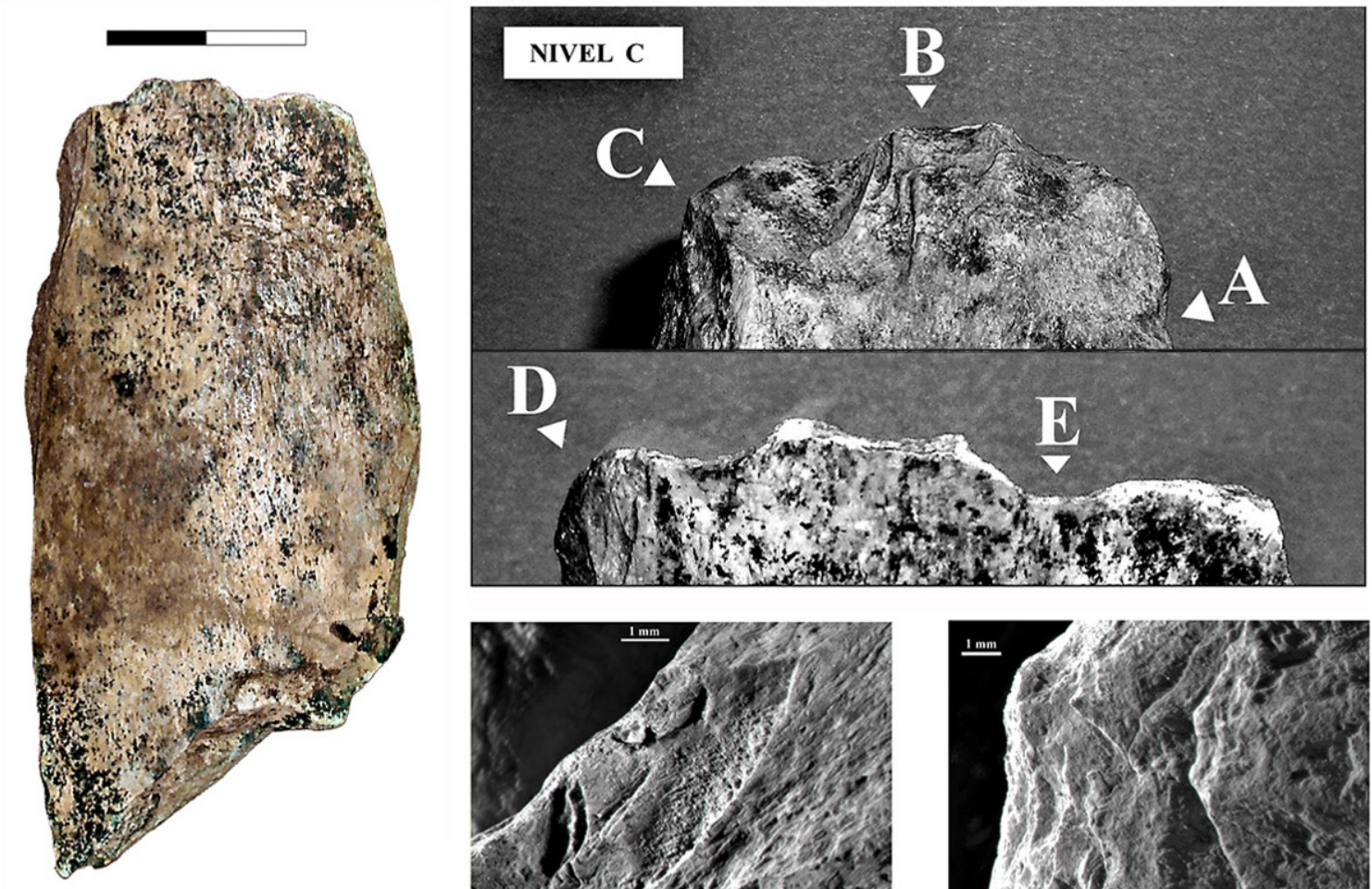

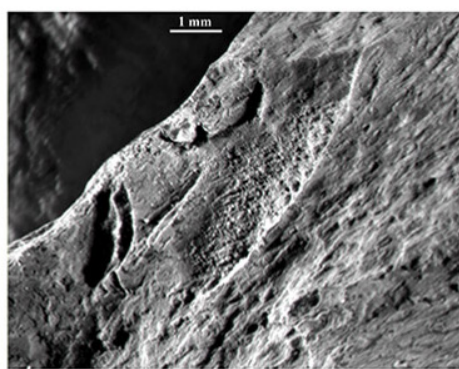

A

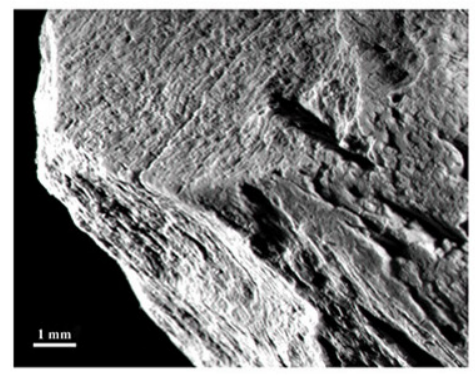

C

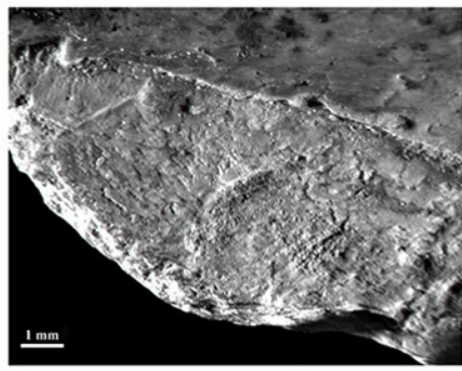

D

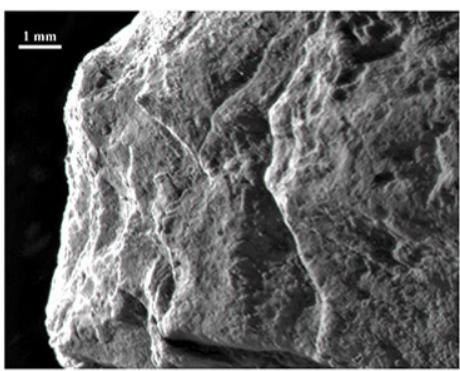

B

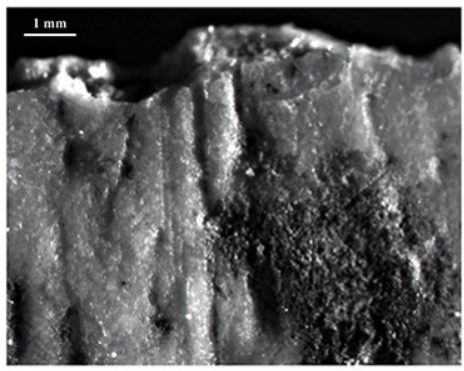

E

Figura 5.- Útil doble (retocador y cincel) de Axlor Nivel C, con detalle del uso como cincel para trabajar una materia dura (i. e. madera o hueso). A partir de Figuras 2 a 7 en Mozota (2008).

de Gönnersdorf (11 ejemplares) y Andernach (10 ejemplares) muy similares a los musterienses. También en el Magdaleniense de Isturitz (SaintPérier 1930), y en el de Labastide (Rigaud 2007).

En el continente americano, sobre todo en su mitad sur, también se ha documentado la presencia de retocadores óseos en diversos contextos de cazadores-recolectores. Es de particular interés el tipo definido por el investigador Jackson (1990) como "retocadores extremo-laterales", que es prácticamente idéntico al concepto de retocador sobre esquirla diafisaria, usado en percusión, que se documenta en los yacimientos musterienses europeos. Esos "retocadores extremo-laterales" se han hallado en contextos paleo-indios de la etapa arcaica (Pleistoceno) en Magallanes, en Fell 1 (Massone y Prieto 2004), y Cueva Lago Sofía 1 (Jackson y Prieto 2005). Para el periodo reciente (Holoceno) se conocen retocadores extremolaterales también en Magallanes, en el sitio de Orejas de Burro 1 (Lorena-L'Heureux 2008). 

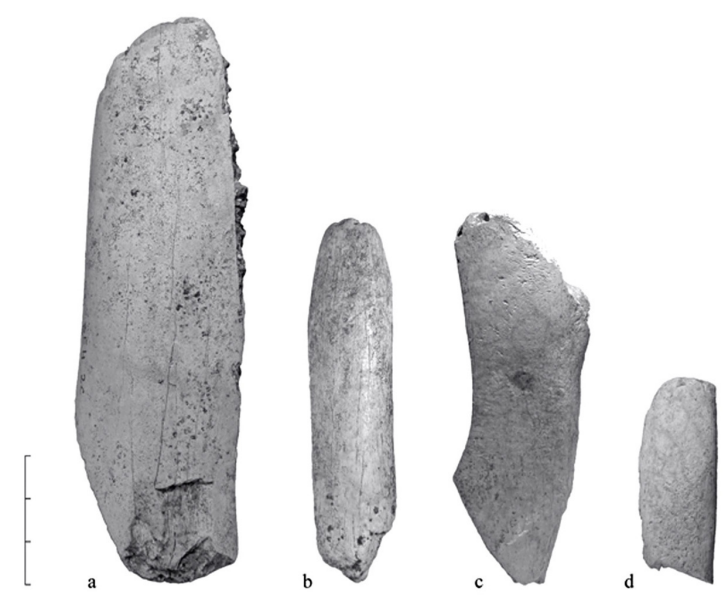

Figura 6.- Cinceles o "pièces intermédiaires" (fr.) del Auriñaciense de la Grotte des Hyènes. Los objetos marcados como "c" y "d" son también retocadores. A partir de Figura 1 en Tartar (2003).

\section{Conclusiones}

Una primera cuestión, constatada en la revisión de las evidencias del Paleolítico inferior y medio, es que el uso del hueso como utillaje está presente desde los momentos más antiguos de la aparición del género Homo.

Por otro lado, a pesar de que no se desarrolle un utillaje óseo al estilo del Paleolítico superior, la obtención y modificación de la materia ósea para su uso es una constante en el Paleolítico medio (y algo esporádico en el Paleolítico inferior). Ese recurso a la materia ósea por parte de las poblaciones del Paleolítico medio resulta obvio al considerar el gran conjunto de evidencias que vienen siendo documentadas desde los inicios de la investigación sobre Prehistoria. Sin embargo, y como hemos visto, la apariencia de esos materiales y las inercias de la investigación han limitado el alcance de las aportaciones, y han minimizado su papel en la reconstrucción de los modos de vida y los procesos históricos de las sociedades humanas.

Además, ese utillaje "poco elaborado" está muy presente a lo largo de todo el Paleolítico superior y también en contextos holocenos. Una vez más, la relativa falta de atención a esas evidencias ha motivado que, salvo excepciones (Tartar 2012), no formen parte sustancial de las explicaciones sobre los modos de vida, ni se tengan en cuenta para proponer modelos de desarrollo histórico de las sociedades humanas.

Otra constatación es que las destrezas técnicas (y las capacidades cognitivas) para modificar la materia ósea al modo del Paleolítico superior están plenamente presentes en el Paleolítico medio -y al menos parcialmente, en el Paleolítico inferior.

Para el Paleolítico medio, esto incluye la capacidad de obtener una morfología concreta usando tanto técnicas de talla (percusión) como de raspadoy abrasión del hueso. Y también la capacidad de planificar y llevar a cabo procesos productivos complejos, que implican una importante inversión de trabajo, y la sucesión y combinación de distintas técnicas y procedimientos.

En lo que se refiere por tanto a las "industrias óseas" (sensu Patou-Mathis 1999) las principales diferencias con el Paleolítico superior se limitan a lo cuantitativo y a la amplitud cronológica y geográfica de las evidencias. Es decir, es una cuestión de frecuencia y ubicuidad, y no puede plantearse en términos de ausencia o presencia absolutas.

Para el Paleolítico inferior, se documentan algunas técnicas típicas de la fabricación de utillaje óseo en momentos posteriores (como modificar la materia ósea por abrasión, para confeccionar un útil determinado) en momentos muy tempranos -entre los primeros representantes del género Homo.

Retomando la cuestión de las incapacidades de los llamados humanos arcaicos (e. g. Homo neanderthalensis), en primer lugar cabe insistir en que la modificación del hueso mediante procesos complejos y planificados está probada en varios contextos (Gaudzinski 1999, Auguste 2002) asociados a esas poblaciones. Además, es relevante que, al aplicar la definición de industria (Patou-Mathis 1999) al Paleolítico inferior y medio, se documente: (1) presencia manifiesta de "industrias líticas", de trabajo de la piedra tallada; y (2) ausencia relativa de tales manifestaciones sobre hueso (en el sentido de utillaje elaborado con gran inversión de trabajo, una cadena operativa compleja, y dimensiones repetidas y estandarizadas).

No obstante la materia ósea se utiliza para obtener otros tipos de útiles. Y, como se ha explicado, se conocen las técnicas básicas de producción de utillaje óseo que serán usadas en el Paleolítico superior. Estas observaciones sugieren que no se trata de una cuestión de capacidades o incapacidades cognitivas. Y por ello, no hay argumentos de peso para explicar la aparición del utillaje óseo del inicio del Paleolítico superior en términos de inferioridad cognitiva de una población europea nativa (Neandertales) frente otra inmigrante o invasora (HAM).

Esto, a su vez, permite plantear que la aparición de ese utillaje óseo de inicios del Paleolítico superior puede ser consecuencia de otros factores. 
En particular, sugiere que dicha irrupción de diferentes tipos de utillaje puede estar relacionada con cambios en la estructura social y económica de esos grupos. Es decir, como parte de los cambios históricos experimentados por unas y otras poblaciones.

A ese respecto, es interesante señalar que las últimas poblaciones neandertales generaron dos culturas arqueológicas del Paleolítico superior inicial: el Chatelperroniense (Caron et al. 2012) en la actual Francia y el Cantábrico, y el Uluzziense (d’Errico et al. 2012) en la Península Itálica aunque también se ha sugerido una filiación HAM de este tecnocomplejo (Benazzi et al. 2011). En ambos casos han librado un utillaje óseo al modo del Paleolítico superior.

Es relevante, además, que dichas culturas arqueológicas sean inmediatamente anteriores (Jöris et al. 2011), o se yuxtapongan en cronología, a las evidencias asociadas a los primeros HAM en Europa, y en particular al primer Auriñaciense. Con todas las reservas que merece lo parco del registro de esa etapa, las evidencias disponibles sugieren que pudieron existir dinámicas variables, complejas, no lineales, $\mathrm{y}$ al largo plazo (esto es, abarcando varios miles de años y una escala continental) de interacción entre esas poblaciones que han sido definidas en términos paleontológicos y genéticos (i.e. $H$. neanderthalensis y $H$. sapiens), y en términos de culturas arqueológicas (Chatelperroniense, Protoauriñaciense, Auriñaciense, etc).

Esta posibilidad reforzaría la idea de que la aparición generalizada del utillaje óseo típico del
Paleolítico superior debe buscarse en las dinámicas históricas: En cambios estructurales (en términos sociales y económicos) que tuvieron lugar en las sociedades de cazadores-recolectores de ese periodo, y que generan unas nuevas necesidades y condicionantes en la producción del utillaje.

A la luz de las evidencias recogidas por un siglo y medio de historiografía, esas necesidades $\mathrm{y}$ condicionantes parecen centrarse en la formalización y estandarización de las técnicas de producción de una parte del utillaje óseo (y no todo, como hemos visto).

Eso supone una inversión mucho mayor de tiempo y esfuerzo en los procesos productivos de dichos útiles. A esas cualidades se yuxtapone, con posterioridad, la incorporación de elementos simbólicos (decoración). Para el autor de este trabajo, esos elementos (que aparecen entre los algunos grupos neandertales, y en los primeros HAM), se deben no tanto a cuestiones de eficiencia o innovación tecnológica, sino al papel socioeconómico más amplio que cumplen esos útiles en concreto, en el seno las sociedades que los producen.

Dicho de otro modo: el trabajo adicional invertido en la fabricación, formalización tipométrica y decoración implican cambios en la apreciación y valoración social de una parte de los instrumentos de trabajo. El aportar una hipótesis suficientemente consistente para explicar esos cambios escapa al alcance de este trabajo, ya que implicaría una revisión exhaustiva y analítica de las evidencias del Paleolítico superior inicial, para compararlas con las de etapas anteriores.

\section{REFERENCIAS BIBLIOGRÁFICAS}

Aguirre, E. (2005/2006): La industria ósea primitiva de Torralba. Munibe (Antropologia-Arkeologia), 57 (1): 19-52. [URL: www.euskomedia.org/PDFAnlt/munibe/aa/200502019052.pdf ]. [Actualizada el 01/04/2012]. Acceso el 21/03/2013.

Ahern J.; Karavanic, I.; Paunovic, M.; Jankovic, I.; Smith, F. (2004): New discoveries and interpretations of hominid fossils and artifacts from Vindija cave, Croatia. Journal of Human Evolution, 46: 27- 67. http://dx.doi. org/10.1016/j.jhevol.2003.09.010

Anzidei, A.P. (2001): Tools from elephant bones at La Polledrara di Cecanibbio and Rebibbia-Casal de' Pazzi. La terra degli Elefanti. Actas del $1^{\circ}$ Congreso Internacional. Roma 16-20 octubre 2001 (G. Cavarretta, P. Gioia, M. Mussi, M.R. Palombo, eds.), Consiglio Nazionale delle Ricerche, Roma: 415-418.

Anzidei, A.P.; Biddittu, I.; Gioia, P; Muss, M.; Piperno, M. (2001): Lithic and bone industries of OIS 9 and OIS 7 in the Roman area. La terra degli Elefanti. Actas del $1^{\circ}$ Congreso Internacional. Roma 16-20 octubre 2001 (G. Cavarretta, P. Gioia, M. Mussi, M.R. Palombo, eds.), Consiglio Nazionale delle Ricerche, Roma: 3-9.

Antunes, M.T. (2000): Bone implements, modified bones and teeth (late Mousterian) from Gruta da Figueira Brava (Arrábida, Portugal). Memórias da Academia das Ciências de Lisboa, Classe de Ciências, 28: 339-379.

Armand, D.; Delagnes, A. (1998): Les retouchoirs en os d'Artenac (couche 6c): perspectives archéozoologiques, taphonomiques et expérimentales. Actes des XVIIIe Rencontres internacionales d'archéologie et d'histoire 
d'Antibes, 1997; Économie préhistorique: les comportements de subsistance au Paléolithique (J.P. Brugal, L. Meignen, M. Pathou-Mathis, eds.), APDCA, Sophia Antipolis: 205-214.

Auguste, P. (2002): Fiche d'éclats diaphysaires du Paléolithique moyer: Biache-Saint-Vaast (Pas-de-Calais) et Kulna (Moravie, République tchèque). En Patou-Mathis 2002: 39-57.

Barandiarán, I. (1987): Manipulación y uso de restos óseos. La cueva de Peña Miel. Nieva de Cameros, La Rioja (P. Utrilla, J. Vilchez, L. Montes, I. Barandiarán, J. Altuna, E. Gil, P. López, eds.), Ministerio de Cultura, Madrid: 87-101.

Baudoin, M. (1906): Discussion sur l'usage de l'os comme outil à l'époque moustérienne. Bulletin de la Société préhistorique de France, 3: 189-200.

Beauval, C. (2004): La faune des niveaux mousteriens de "Chez-Pinaud" (Jonzac, Charente-Maritime, France. Prémiere analyse. Le site paléolithique de Chez-Pinaud à Jonzac, Charente Maritime (J. Airvaux, ed.), Association Préhistoire du sud-ouest, Cressensac: 125-156.

Benazzi, S.; Douka, K.; Fornai, C.; Bauer, C.C.; Kullmer, O.; Svoboda, J.; Pap, I.; Mallegni, F.; Bayle, P.; Coquerelle, M.; Condemi, S.; Ronchitelli, A.; Harvati, K.; Weber K.W. (2012): Early dispersal of modern humans in Europe and implications for Neanderthal behaviour. Nature, 479: 525-528. http://dx.doi. org/10.1038/nature10617

Bermúdez de Castro, J.M.; SÁenz de Buruaga, A. (1999): Étude préliminaire du site pléistocène supérieur à hominidé d'Arrillor (Pays Basque, Espagne). L'Anthropologie, 103 (4): 633-639.

Binford, L. (1981): Bones: Ancient Men and Modern Myths. Academic Press, Nueva York.

BINFORD, L. (1983): Working at Archaeology. Academic Press, Nueva York.

Boeda, E.; Bourguignon, L.; Griggo, C. (1998): Activités de subsistance dans la couche Vi3b' (Moustérien) du gisement d'Umm El Tlel (Syrie). Actes des XVIIIe Rencontres internacionales d'archéologie et d'histoire d'Antibes, 1997; Économie préhistorique: les comportements de subsistance au Paléolithique (J.P. Brugal, L. Meignen, M. Pathou-Mathis, eds.), APDCA, Sophia Antipolis: 243-258.

Bordes, F. (1961): Typologie du Paleolithique ancien et moyen. CNRS, Paris.

BOURGUIGNON, L. (2001): Apports de l'experimentation et de l'analyse techno-morfo-fonctionelle à la reconnaisance de processus d'amenagement de la retouche Quina. Prehistoire et approche expérimentale; Prehistoire, 5: 3566.

BraIN, C.K. (1981): The Hunters Or the Hunted?: An Introduction to African Cave Taphonomy. University of Chicago Press, Chicago.

Breuil, H.; Obermaier, H. (1914): Institut de Paléontologie humaine. Travaux de l'année 1913, L'Anthropologie, 25: 233-253

Caron, F.; D’Errico, F.; Del Moral, P., Santos, F.; Zilhão, J. (2011): The reality of Neandertal symbolic behavior at the Grotte du Renne, Arcy-sur-Cure, France. PloS one, 6 (6): 215-245. http://dx.doi.org/10.1371/journal. pone. 0021545

Castel, J.C.; Chauvière, F.X.; L'Homme, X.; Camus, H. (2006): Un nouveau gisement du Paléolithique supérieur récent: le Petit Cloup Barrat (Cabrerets, Lot, France). Bulletin de la Société préhistorique française, 103 (2): 263-273.

Castel, J.C.; Madelaine, S. (2006): Quelques éléments remarquables de la faune du Solutréen de LaugerieHaute (Les-Eyzies-de-Tayac, Dordogne). Paléo 18: 275-284 [URL http://paleo.revues.org/258 ]. [Actualizada el 23/04/2009]. Acceso el 21/03/2013.

Chase, P.G. (1990): Tool-making tools and middle Paleolithic behaviour. Current Anthropology, 31: 443-447.

Chernysh, A.P. (1982): Paleolithic multilayer Molodova I site (in Russian). Molodova I: Unique Mousterian settlement in the middle Dniestr region (G.I. Goretsky, I.K. Ivanova, eds.), Nauka, Moscú: 6-102.

CostA, A.G. (2010): A geometric morphometric assessment of plan shape in bone and stone Acheulean bifaces from the Middle Pleistocene site of Castel di Guido, Latium, Italy. New perspectives on old stones: analytical approaches to Paleolithic technologies (S.J. Lycett, P.R. Chauhan, eds.), Springer, Nueva York: 23-41. http:// dx.doi.org/10.1007/978-1-4419-6861-6_2

Daleau, F. (1874): Grotte des Fees (âge du renne), situeè au Roc, commune de Marcamps, canton Bourg (Gironde). Memoires de la Société Archeologique de Bordeaux, 1: 109-119.

DART, R. (1957): The osteodontokeratic culture of Australopithecus prometheus, Memoir (Transvaal Museum), 10: $1-105$.

Dauvors, M. (1974): Industrie osseuse préhistorique et expérimentation. ler Colloque sur l'industrie de l'os dans 
la Préhistoire (H. Camps-Fabrer, ed.), Université de Provence, Paris: 73-88.

David, F.; Connet, N.; Girard, M.; Miskovsky, J.C.; Mourer-Chauviré, C.; Roblin-Jouve, A. (2005): Les niveaux du Paléolithique supérieur à la grotte du Bison (Arcy-sur-Cure, Yonne): couches a à d. Revue archéologique de l'Est, 54: 5-50.

Demay, L.; Péan, S.; Patou-Mathis, M. (2012): Mammoths used as food and building resources by Neanderthals: Zooarchaeological study applied to layer 4, Molodova I (Ukraine). Quaternary International, 276-277: 212226 http://dx.doi.org/10.1016/j.quaint.2011.11.019

D'Errico, F. (2007): De Swartkrans a Arcy-sur-Cure, el uso de instrumentos óseos en el Paleolítico Inferior y Medio. El Universo Neandertal I (E. Baquedano, ed.), F. D. S. -Ibersaf, Soria: 122-133.

D'Errico, F.; Backwell L.R. (2003): Possible Evidence of Bone Tool Shaping by Swartkrans Early Hominids. Journal of Archaeological Science, 30 (12): 1559-1576. http://dx.doi.org/10.1016/S0305-4403(03)00052-9

D'Errico, F.; Borgia, V.; Ronchitelli, A. (2012): Uluzzian bone technology and its implications for the origin of behavioural modernity. Quaternary International, 259: 59-71. http://dx.doi.org/10.1016/j.quaint.2011.03.039

D'Errico, F.; Giacobini, G. (1988): L'apport des études des surfaces pour l'interprétation des modifications sur l'os au Paléolithique moyen. L'Homme de Neandertal, La Technique (M. Otte, ed.), ERAUL 35, Lieja: 39-47.

D'Errico, F.; Villa, P. (1997): Holes and grooves: the contribution of microscopy and taphonomy to the problem of art origins, Journal of Human Evolution, 33 (1): 1-31. http://dx.doi.org/10.1006/jhev.1997.0141

Díez-Fernández Lomana, C.; Navazo, M. (2005): Apuntes sociales y geográficos a partir de los yacimientos del Paleolítico Medio en la zona nororiental de la Meseta castellano leonesa. Neandertales Cantábricos. Estado de la cuestión (R. Montes, J.A. Lasheras, eds.), Museo de Altamira, Madrid: 39-54.

Dobosi, V.T. (2001): Ex Proboscideis-Proboscidean remains as raw material at four Palaeolithic sites, Hungary. La terra degli Elefanti. Actas del $1^{\circ}$ Congreso Internacional. Roma 16-20 octubre 2001 (G. Cavarretta, P. Gioia, M. Mussi, M.R. Palombo, eds.), Consiglio Nazionale delle Ricerche, Roma: 429-431.

Filipov, A.K.; Lioubine, V.P. (1993): La grotte de Barakaevskaïa (Caucase du Nord): Les Retouchoirs osseux et la planigraphie des vestiges culturels. L'Anthropologie, 97: 299-310.

Freeman, L.G. (1971): El hueso trabajado musteriense de Cueva Morín. Cueva Morín. Excavaciones. 19661968 (J. González Echegaray; L.G. Freeman, eds.), Patronato de las Cuevas Prehistóricas de la Provincia de Santander. Santander: 135-159.

Gaudzinski, S. (1999): Middle Palaeolithic Bone Tools from the Open-Air Site Salzgitter-Lebenstedt (Germany). Journal of Archaeological Science, 26 (2): 125-141. http://dx.doi.org/10.1006/jasc.1998.0311

González Urquijo, J.; Ibáñez Estévez, J.J.; Rios-Garaizar, J. Bourguignon, L.; Castaños Ugarte, P., Tarriño, A. (2005): Excavaciones recientes en Axlor. Movilidad y planificación de actividades en grupos de neandertales. Neandertales Cantábricos. Estado de la cuestión (R. Montes, J.A. Lasheras, eds.), Museo de Altamira, Madrid: 527-539.

HenRi-Martin, L. (1906): Maillets ou enclumes en os provenant de la couche moustérienne de la Quina (Charente). Bulletin de la Société Préhistorique Française, 3: 155-162.

Henshilwood, C.S.; D’Errico, F.; Marean, C.W.; Milo, R.G.; Yates, R. (2001): An early bone tool industry from the Middle Stone Age at Blombos Cave, South Africa: implications for the origins of modern human behaviour, symbolism and language. Journal of Human Evolution, 41: 631-678. http://dx.doi.org/10.1006/ jhev.2001.0515

JACKSON, D. (1990): Retocadores extremo-laterales en contextos Paleo-indios. Anales del Instituto de la Patagonia, Serie Ciencias Sociales, 19: 121-124.

JACKson, D.; Prieto, A. (2005): Estrategias tecnológicas y conjunto lítico del contexto paleoindio de Cueva Lago Sofía 1, Última Esperanza, Magallanes. Magallania, 33 (1): 115-120. http://dx.doi.org/10.4067/S071822442005000100008

JÉQUIER, C.A.; RomANDINI, M.; PeRESANI, M. (2012): Les retouchoirs en matières dures animales: une comparaison entre Moustérien final et Uluzzien. Comptes Rendus Palevol, 11 (4): 283-292. http://dx.doi.org/10.1016/j. crpv.2011.12.001

Jöris, O.; Street, M.; Terberger, T.; Weninger, B. (2011): Radiocarbon Dating the Middle to Upper Palaeolithic Transition: The Demise of the Last Neanderthals and the First Appearance of Anatomically Modern Humans in Europe. Vertebrate Continuity and Discontinuity in the Peopling of Europe One Hundred Fifty Years of Neanderthal Study, Paleobiology and Paleoanthropology (S. Condemi, G.-C. Weniger, eds.), Springer, Berlin: 239-298. 
Kolen, J. (1990): Hominids without homes: on the nature of Middle Paleolithic settlement in Europe. The Middle Paleolithic Occupation of Europe (W. Roebroeks, C. Gamble, eds.), University of Leiden Press, Leiden: 139175.

Kozlowski, J.K. (1982): Excavation in the Bacho Kiro cave (Bulgaria): final report. Panstwowe Wydawnictwo Naukowe, Warzawa.

Leroy-Prost, C. (1974): L'industrie osseuse aurignacienne de la collection Passemard d'Isturizt (PyrénéesAtlantiques). L'Anthropologie, 78: 283-297.

Leroy-Prost, C. (2002): Fiche Canines de Carnivores. En Patou-Mathis 2002: 99-104.

LEONARDi, P. (1979): Una serie di ritoccatoi prevalentemente musteriani del Riparo Tagliente in Valpantena presso Verona, Prehistoria alpina, 15: 7-15.

Lorena-L'Heureux, G. (2008): La arqueofauna del Campo Volcánico Pali Aike. El sitio Orejas de Burro 1, Santa Cruz, Argentina. Magallania, 36 (1): 65-78. http://dx.doi.org/10.4067/S0718-22442008000100006

Malerba, G.; Giacobini, G. (1996): Les Retouchoirs sur éclats diaphysaires du Paléolitique moyen et supérieur de l'abri de Fumane et de la grotte de San Bernardino (Vénétie, Italia Nord Orientale). Abstracts, XIII internacional congress of UISPP. Forli (C. Peretto, C. Giunchi, eds.), A.B.A.C.O. Edizioni, Roma: 167-171.

Martínez-Moreno, J. (2005): Comportamientos y tecnologías polémicas: las industrias en hueso "poco elaboradas" y objetos simbólicos del Paleolítico Medio Cantábrico. Neandertales Cantábricos. Estado de la cuestión (R. Montes; J.A. Lasheras, eds.), Museo de Altamira, Madrid: 349-367.

Massone, M.; Prieto, A. (2004): Evaluación de la modalidad Fell 1 en Magallanes. Chungará. Revista Chilena de Antropología, número especial: 303-315.

Mellars, P. (1991): Cognitive changes and the emergence of modern humans in Europe. Cambridge Archaeological Journal, 1 (1): 63-76. http://dx.doi.org/10.1017/S0959774300000251

Mellars, P. (2004): Neanderthals and the modern human colonization of Europe. Nature, 432: 461-465. http:// dx.doi.org/ 10.1038/nature03103

Michel, P.; Campmas, E.; Stoetzel, E.; Nespoulet, R.; Abdelualil El Hajraoui, M.; Amani, F. (2009): La macrofaune du Pléistocène supérieur d'El Harhoura 2 (Témara, Maroc): données préliminaires, L'Anthropologie, 113: 283-312.

Moncel, M.H.; Moigne, A.M.; Combier, J. (2005): Pre-Neandertal behaviour during isotopic stage 9 and the beginning of stage 8 . New data concerning fauna and lithics in the different occupation levels of orgnac 3 (Ardèche, South-East France): occupation types. Journal of Archaeological Science, 32 (9): 1283-1301. http:// dx.doi.org/10.1016/j.jas.2005.03.014

Mortillet, G.; Mortillet, H. (1910): La Préhistoire-Origine et Antiquité de l'Homme. Schleicher, Paris.

Mozota, M. (2007): Industrias óseas musterienses en el Cantábrico oriental: los "alisadores" en hueso de los niveles B, C y D de Axlor (Dima, Bizkaia). Cuadernos de Arqueología de la Universidad de Navarra, 15: 31-42. [URL http://dspace.unav.es/dspace/handle/10171/8910 ]. [Actualizada el 09/08/2010]. Acceso el $21 / 03 / 2013$.

Mozota, M. (2008): Estudio tafonómico y tecnológico de un útil doble "cincel/retocador", proveniente del nivel C (Musteriense) de Axlor - Dima, Bizkaia. Zephyrus, 61: 217-224. [URL http://campus.usal.es/ revistas_trabajo/ index.php/0514-7336/article/viewFile/1082/1160 ] [Actualizada el 10-06-2011]. Acceso el 21/03/2013.

Mozota, M. (2009): El utillaje óseo Musteriense del nivel "D" de Axlor (Dima, Vizcaya): análisis de la cadena operativa. Trabajos de Prehistoria, 66 (1): 28-46. http://dx.doi.org/10.3989/tp.2009.09011

Mozota, M. (2012): El hueso como materia prima: El utillaje óseo del final del Musteriense en el sector central del norte de la Península Ibérica. Tesis Doctoral. Universidad de Cantabria. [URL: www.educacion.gob.es/ teseo/imprimirFicheroTesis.do?fichero=34920 ]. [Actualizada el 16/10/2012]. Acceso el 20/03/2013.

Noble, W.; Davidson, I. (1996): Human Evolution, Language and Mind: A Psychological and Archaeological Inquiry. Cambridge Univ. Press, Cambridge.

Passemard, E. (1922): La Caverne d'Isturitz (BassesPyrénées). Revue Archéologique, 15: 1-45.

Patou-Mathis, M. (1999): Les outils osseux du Paléolithique inférieur et moyen en Europe. Problèmes, méthodes et résultats préliminaires. Préhistoire d'os. Recueil d'études sur l'industrie osseuse préhistorique offert à Henriette Camps-Fabrer, Publications de l'Université de Provence, Aix-en-Provence: 49-57.

Patou-Mathis, M. (ed.) (2002): Fiches typologiques de l'industrie osseuse préhistorique. Cahier X: Retouchoirs, compresseurs, percuteurs. Os à impressions et à eraillures. Société Préhistorique Française, Paris.

Patou-Mathis, M. (2007): Analyses Archéozoologiques des Unités III et IV de Kabazi V. Kabazi V: Interstratification 
of Micoquian \& Levallois-Mousterian Camp Sites. Palaeolithic Sites of Crimea (V.P. Chabai, J. Richter, Uthmeier, eds.), Simferopol-Cologne, Shlyakh: 79-128.

Peresani, M.; Fiore, I.; Gala, M.; Romandini, M.; Tagliacozzo, M. (2011): Late Neandertals and the intentional removal of feathers as evidenced from bird bone taphonomy at Fumane Cave 44 ky B.P., Italy. PNAS, 108 (10): 3888-3893. http://dx.doi.org/10.1073/pnas.1016212108

Peyrony, D. (1934): La Ferrasie. Préhistorie, 3: 1-92.

Radmilli, A.M.; Boschian, G. (1996): Gli scavi a Castel di Guido, il piu antico giacimento di cacciatore del Paleolitico inferiore nell'Agro Romano. Istituto Italiano di Preistoria e Protostoria, Firenze.

Raynal, J.P.; Le Corre-Le Beux, M.; Santagata, C.; Fernandes, P.; Guadelli, J.L.; Fiore, I.; Tagliacozzo, A.; Lemorini C.; Rhodes E.J.; Bertran P.; Kieffer G.; Vivent D. (2005): Paleolithique Moyen dans le Su du Massif Central: Les Donees du Velay (Haute-Loire, France). Données récentes sur les modalités de peuplement et sur le cadre chronostratigraphique, géologique et paléogéographique des industries du Paléolithique inférieur et moyen en Europe - Rennes, 22-25 septembre 2003 (N. Molines, M.H. Moncel, J.L. Monnier, eds.), BAR international series, Oxford: 173-201.

Raynal, J.P.; Santagata, C.; Deaujard, C.; Fernandes, P.; Guadelli, J.-L.; Kiefer, G. (2010): Fouille programmée annuelle. Polignac. Site de la grotte de Sainte-Anne 1. Bilan scientifique de la Région Auvergne, 2008, Ministère de la Culture et de la Communication, Paris: 83-86.

Rigaud, A. (2007): Retouchoirs sur éclats diaphysaires ou « affûtoirs » de Labastide (Hautes-Pyrénées): Du barbarisme scientifique à la rigueur artisanale au travers de l'expérimentation. Archéologie des Pyrénées occidentales et des Landes, 26: 193-200.

Rios-Garaizar, J. (2008): Variabilidad tecnológica en el Paleolítico Medio de los Pirineos Occidentales: una expresión de las dinámicas históricas de las sociedades neandertales. Treballs de Arqueologia, 14: $171-194$. [URL http://ddd.uab.cat/pub/tda/11349263n14p171.pdf] [Actualizada el 04/12/2012]. Acceso el 20/03/2013.

Rios-Garaizar, J. (2012): Técnicas de caza en el Paleolítico Medio del País Vasco. Isturitz, 12: 7-37.

Roberts, M.B.; Parfitt, S.A. (1999): Boxgrove: A Middle Pleistocene Hominid Site at Eartham Quarry, Boxgrove, West Sussex. English Heritage, London.

Rosell, J.; Blasco, R.; Campeny, G.; Diez, J.C.; Alonso Alcalde, R.; Menendez, L.; Arsuaga, J.L.; Bermudez de Castro, J.M.; Carbonell, E. (2011): Bone as a technological raw material at the Gran Dolina site (Sierra de Atapuerca, Burgos, Spain). Journal of Human Evolution, 61 (1): 125-131. http://dx.doi.org/10.1016/j. jhevol.2011.02.001

Sáenz de Buruaga, A. (1987): Aplicación de la tipología analítica al estudio del comienzo del Paleolítico superior en Euskalherria: El caso de la Cueva de Gatzarria (Zuberoa). Tesis Doctoral, Universidad del País Vasco.

SÁenz de Buruaga, A. (1996): Acerca del posible y derivado carácter tecnológico de ciertos tipos óseos debitados los casos de muescas, "écaillés” y retocadores. Veleia: Revista de prehistoria, historia antigua, arqueología y filología clásicas, 13: 71-76.

SAINT-PÉRIER, R. DE (1930): La grotte d'Isturitz I-Le Magdalénien de la Salle de Saint-Martin. Masson et Cie, Paris.

SAnguino, J.; Montes, R. (2005): Nuevos datos para el conocimiento del Paleolítico Medio en el centro de la Región Cantábrica: La Cueva de Covalejos. Neandertales Cantábricos. Estado de la cuestión (R. Montes; J.A. Lasheras, eds.), Museo de Altamira, Madrid: 489-538

Schwab, C. (2003): Les os à impressions et à éraillures de la grotte d'Isturitz (Pyrénées Atlantiques, France). L'industrie osseuse pré- et protohistorique en Europe: approches technologiques et fonctionnelles. Actes du colloque 1.6, XIV congrès de l'UISPP, Liège, 2-8/09/2001 (M. Patou-Mathis, P. Catelain, D. Ramseyer, eds.), Cercle archéologique Hesbaye-Condroz, Armay: 9-18.

Segre, A.G.; Ascenzi, A. (1984): Fontana Ranuccio: Italy's earliest middle pleistocene hominid site. Current Anthropology, 25: 230-233.

Semenov, S.A. (1956): Retouchoirs de pierre du Paléolitique récent. Annales du Centre d'Études et de Documentation Paléontologique, 18: 382-387.

Siret, L. (1925): L'emploi de l'os dans la retouche des silex moustériens. Bulletin de la Société Préhistorique Française, 22: 208-210.

Stephanchuk, V.N. (1993): Prolom II, a Middle Palaeolithic cave site in the eastern Crimea with non utilitarian bone artefacts. Proceedings of the Prehistoric Society, 59: 17-37.

Straus, L.G. (1992): Iberia before the Iberians. University of New Mexico Press, Albuquerque. 
TARTAR, E. (2003): Transformation et utilisation préhistoriques des matières osseuses: L'analyse technofonctionnelle del'industrie en matières osseuses dite 'peu élaborée': L'exemple des pièces intermédiaires en os de l'Aurignacien ancien de la grotte des Hyènes (Brassempouy, Landes). Préhistoire anthropologie méditerranéennes, 12: 139146.

TARTAR, E. (2012): The recognition of a new type of bone tools in Early Aurignacian assemblages: implications for understanding the appearance of osseous technology in Europe. Journal of Archaeological Science, 39 (7): 2348-2360. http://dx.doi.org/10.1016/j.jas.2012.02.003

TAuTE, W. (1965): Retoucheure asu Knochen, Zahnbein und Stein vom Mittelpaläolithikum vis zum Neolithikum. Fundberichte aus Schawaben, 17: 76-102.

Thenius, E. (1973): Fossils and the Life of the past. The English Universities Press Ltd., London.

TinNEs, J. (2001): Die retuscheure aus Knochen der Magdalénien-Fundplätze Gönnersdorf und Andernach. ZeitRäume, Gedenkschrift für Wolfgang Taute (W. Taute, B. Gehlen, A. Tillmann, M. Heinen, eds.), Deutsche Gesellschaft für Ur- und Frühgeschichte, Bonn: 337-347.

VAlEnsi, P. (1996): Taphonomie des grands mamifères et palethnologie á la grotte du Lazaret (Nice, France). Anthropozoologica, 3: 13-28.

VALENSI, P. (2002): Fiche extrémités distales d'humérus de grands ongulés. En Patou-Mathis 2002: 29-37.

Valoch, K. (1988): Die Erforshung der Kulna-Hïle 1961-1976. Anthropos, 24 (16). Número especial.

Verna, C.; D'Errico, F. (2011): The oldest evidence for the use of human bone as tool. Journal of human evolution, 60: 145-157. http://dx.doi.org/10.1016/j.jhevol.2010.07.027

Vincent, A. (1985): Préliminaires expérimentaux du façonnage de l'os par percussion directe. Quelques reproductions d'artefacts reconnus dans les niveaux du Paléolithique moyen. Outillage peu élaboré en os et en bois de cervidés (artefacts I) (M. Patou-Mathis, ed.), Editions du CEDA, Vironval: 23-32.

Vincent, A. (1987): Outillage osseux du Paléolithique moyen à Bois Roche (Cherves-Richemont, Charente)? Etude préliminaire. Préhistoire de Poitou-Charente, Problèmes Actuels. Actes du IIIe Congrès national des sociétés savantes, Poitiers 1986 (C.T.H.S., eds.), Ministère de l'éducation national, Paris: 201-206.

Vincent, A. (1988): L'os comme artefact au Paleolithique Moyen: Principes d'etude et premiers resultats. L'Homme de Neandertal, La Technique (M. Otte, ed.), ERAUL 35, Lieja: 185-196.

VIncent, A. (1993): L'outillage osseux au Paléolithique moyen: une nouvelle approche. Tesis Doctoral. Université de Paris-X.

Zilhao, J.; Angelucci, D.E.; Badal-García, E.; D’Errico, F.; Daniel, F.; Dayet, L.; Douka, K., Higham, T.; Martínez-SÁnchez, M.J.; Montes-Bernárdez, R.; Murcia-Mascarós, S.; Pérez- Sirvent, C.; Roldán- García, C.; Vanhaeren, M.; Villaverde, V.; Wood, R.; Zapata, J. (2010): Symbolic use of marine shells and mineral pigments by Iberian Neandertals. PNAS, 107 (3): 1023-1028. http://dx.doi.org/10.1073/pnas.0914088107 